\title{
Mortality in an International Perspective
}

\author{
edited by Jon Anson and Marc Luy \\ New York: Springer 2014 \\ ISBN 978-3-319-03028-9 (print), 978-3-319-03029-6 (online) \\ Hardcover $\$ 179$, e-book $\$ 139,359$ pp.
}

\author{
Reviewed by Sulaiman Bah \\ Department of Health Information Management and Technology, \\ Imam AbdulRahman Alfaisal University, ${ }^{1}$ Dammam, Saudi Arabia
}

The subject of mortality is a meeting ground for diverse disciplines, and hence it is not surprising to see it being approached from remarkably different perspectives. The book has 13 chapters, covering a wide range of topics, using data from different geographic areas. The chapters can be grouped into three broad themes: mortality estimation and projections (chapters 2, 3, and 5), explanation of trends in mortality and causes of death (chapters 4, 8, 9, 11, and 13), and measurement of impact of determinants (chapters 6, 7, 10, and 12).

Chapter 1, by Jon Anson and Marc Luy, adequately summarizes all the chapters in the book. It is clear that the aim of the book is to put together 'state of the art' methods used in mortality and morbidity. However, it is in the chapters dealing with mortality estimation and projections that "cutting edge" methods are used. The remaining chapters, interesting as they are, used methods that would fall under "normal science" rather than cutting-edge methods.

Chapter 2 is authored by Peter Congdon, a pioneer in the analysis of small area mortality and the author of books on Bayesian statistical modelling. In this chapter, he exploited correlations between adjacent ages and areas with Bayesian modelling and applied it to data of over 3,000 US counties. He found that "whereas there is little gain in life expectancy in the lowest income counties, high income counties showed expectancy improvements exceeding the US average." This new approach is an improvement on standard conventional life table methods used in small area mortality that overlook spatial or age correlations.

Chapter 3, by Joroen Spijker, is clearly the most ambitious chapter in the book. He uses data from 21 countries over the period from 1980 to 2000 to model death rates for 11 causes of death. The model used allows for the simulataneous analysis of inter-country and inter-temporal variations in mortality. As a departure from other models based on extrapolation, this model included data on some known socioeconomic determinants of mortality. The model was validated and then used to produce short-term projections of rates due to causes of death. This is a significant contribution in an area that is still in its youthful stage of development.

Chapter 4, by Katalin Kovács, thoroughly reviews the different variants of Epidemiological Transition Theory and the Nutritional Transition Theory. Using causes of death data from Hungary, Kovács tries to group the different causes of death in such a way as to allow her to see the role of the different theories in explaining inequalities in mortality between the less educated and the more educated. Her conclusion was that "nutrition transition theory provides a very plausible explanatory framework for the growth of mortality inequalities."

Chapter 5, by Sarinapha Vasunilashorrn and others, attempts to predict mortality from profiles of biological risk and performance measures of functioning. They were able to get a rich set of data by linking a national US survey data with the causes of death data contained in the National Death Index. According to the authors,

1. Formerly University of Dammam. 
this was the first study "that methodologically examines the relationship of both biomarkers and indicators of functioning using the latent class approach."

Chapter 6, by V. Semoyonova and others, attempts to estimate alcohol-related losses in the Russian population. It is interesting to note that there is marked similarity between the findings in the chapter with those found in some countries affected by high HIV/AIDS-related deaths. In both cases, the underestimation comes from multiple stakeholders, including the state (to avoid negative publicity), next-of-kin (to "save face"), and certifiers and coders (innocently or in collusion with the two aforementioned stakeholders).

Chapter 7, by Maria Pizarro and others, analysed infant mortality in the context of the International Convention of the Rights of the Child (CRC). They show that the main causes of infant deaths could be prevented by simple interventions, and hence, if those causes are addressed, they would help to reduce regional inequalities and hence help Argentina to meet the CRC and related goals. The authors are skeptical about the possibility of a country presenting a "less than truthful" report to the CRC and getting away with it. Indeed, it is not easy for a country to do so, as the CRC allows for NGOs to independently present their own report (alternative report) to the CRC at the same forum that the national committee is presenting its report.

Chapter 8, by İlknur Yüksel-Kaptanoğlu and others, looks at maternal mortality in Turkey. They lament the quality of vital registration data in the country, as well as the conflicting maternal mortality estimates that are published. They try to address these shortcomings by using data obtained from a survey designed after RAMOS (Reproductive Age Mortality Survey). Their research allowed them to obtain estimates of maternal mortality, as well as identify "which avoidable factors are most important for elimination of the maternal deaths in Turkey." The study found that "household and community factors" were the most important of the avoidable factors. This is a surprising finding, given that, as the authors observe "there are no legal barriers to women's participation in education, labour force and political life..."

Chapter 9, by Rosa Gómez Redondo and others, studies Spanish old age mortality over the period 1975 to 2006, using overall and cause- and sex-specific standardized death rates. Based on the reduction in death rates for specific chronic diseases among males, they predict convergence in male and female mortality rates in the future. Such a convergence could result in revision of some of the theories on male-female mortality differentials.

Chapter 10, by Anne Herm and others, asked a very important question: whether or not institutionalization increases or decreases the risk of mortality. Using Hungarian census data, they report, "our results indicate very clearly that, except at very old ages, it is preferable for people to remain at home..." This has major implications for health care provision for the elderly. One policy implication of this finding, at least for Hungary, is the shift in emphasis from nursing homes to home health care. Replication of such a study in other countries will contribute to evidence-based decision making for health care among the elderly.

Chapter 11, by Madelin Gómez León and Esther Maria León Diaz, is on decomposition of the sex differential in life expectancy in Cuba. Close to half of the results section is used to show that "Cuba is in an advanced stage of the demographic transition." But that could have been proven in far fewer pages. Nevertheless, the authors use methods of decomposition of life expectancy by causes of death, and arrive at the conclusion that the four main causes that contribute most to the high sex differential in mortality are heart diseases, external causes of death, cancer, and cerebrovascular disease. If these diseases are addressed, life expectancy could still increase in Cuba.

Chapter 12, by T. Sabgayda and others, addresses the issue of avoidable mortality in Russia. By analyzing data in over 80 Russian regions, they found similar patterns in the spatial variation of avoidable and unavoidable mortality. After doing several comparisons, they reach the conclusion that the "larger increase in unavoidable, compared to avoidable, mortality during the post-Soviet period suggests that the social and political upheavals had a greater influence of the mortality of the Russian population than did the activities of health institutions." This innovative approach at explaining determinants of mortality change is applicable to other areas that have undergone social and political turmoil.

The last chapter, by Zhongwei Zhao and others, looks at long-term mortality changes in East Asia. They look at countries that underwent "rapid mortality transition" (Omran's accelerated model), including, Japan, Hong Kong, and Taiwan. The aim of the chapter is to draw lessons from the experience of those countries. 
According to the authors, the early detection and effective prevention of specific diseases (e.g., neoplasms and CVD) helped them to achieve rapid improvements in mortality over a relatively short period of time.

In all, three chapters dealt with the concept of "preventable/avoidable" causes of death (chapters 7, 8, and 12). All three chapters defined the concept differently. This means that if all three concepts are applied to the same data, different results will be obtained. There is clearly a need for standardization of definitions in this area. In the chapters dealing with causes of death, there is a glaring omission of qualification of the cause of death. Is it a principal cause, underlying cause, or multiple cause? The reader is left to guess. Further, there is no chapter dealing with multiple causes of death or emerging infectious diseases.

While there are no obvious grammatical or typological flaws, there are a few slips. For example, the text makes reference to a blue line on a black and white graph (Fig 11.5) and to green and yellow bars in black and white graphs (Fig 11.12). As usual with similar books published by Springer, there is no list of tables or list of figures.

The book makes a useful contribution to new methods in the field of mortality and morbidity in general, and to the elucidation of trends and determinants. It is recommended for libraries and graduate programs covering the topic of mortality and morbidity. As the scope of chapters is diverse, a researcher interested in only a few of the topics has the option of purchasing only those specific chapters individually from Springer. 